Pensamiento Crítico Vol. 18 № 2, pp. 95-104

\title{
Algunas notas sobre las limitaciones de la Economía Matemática
}

Pablo Rivas Santos

\section{RESUMEN}

El presente Artículo demuestra que el Método matemático para estudiar Economía es un sistema de conocimientos que parte de falsos supuestos y conduce a erróneas conclusiones.

La Estadística de precios es una forma de representar realidades históricas de los Precios. La Estadística de Precios es historia económica; por lo que el Índices de precios son datos de historia económica.

Los economistas matemáticos al abordar la relación de precios y costos ignoran la operación del mercado y pretenden dejar de lado el uso del dinero ( ingrediente del cálculo económico). Sin embargo, implícitamente, suponen la existencia del dinero y su uso; ya que hablan (en general) de precios y costos, pretendiendo confrontar los precios con los costos. Los precios son magnitudes dinerarias; y los costos sólo expresados en términos monetarios entran en el cálculo económico.

Otros economistas matemáticos afirman que cabría basar el cálculo económico en unidades de utilidad.

En Economía advertimos que las personas conscientemente desean provocar cambios. Conocemos las fuerzas que provocan el cambio y tal conocimiento (basado en el razonamiento) nos permite comprender el proceso económico. El 


\section{Pensamiento Crítico Vol. I8. Nº}

economista advierte cuál es eso que impulsa y provoca la aparición del mercado. Gracias a ese conocimiento logra distinguir los fenómenos económicos, de los fenómenos físicos pudiendo, por tal vía, descubrir las leyes rectoras de la actividad del mercado.

En el campo de la Economía Monetaria y la Microeconomía se advierten los negativos efectos del análisis matemático: La "ecuación cuantitativa del dinero" es un estéril y errado intento de abordar el problema de las variaciones del poder adquisitivo del dinero. La afirmación "que los consumidores, al valorar los bienes de consumo, valoran también los factores de producción necesarios para la obtención de esos bienes de consumo" es un falso supuesto para el análisis microeconómico.

La Economía se interesa, directamente por el actuar de las personas. El único cometido de la Ciencia Económica consiste en analizar el actuar de la gente, o sea, en analizar procesos.

Palabras clave: Método matemático, Estadística de precios, Economía, Precios, costos.

\section{ABSTRACT}

This article shows that the mathematical method to study economics is a system of knowledge from false assumptions and leading to erroneous conclusions. The Price Statistics is a way of representing historical realities of Prices. The Statistics of Prices is economic history, so the price indices are economic history data.

Mathematical economists to address the relationship of prices and costs ignore the operation of the market and intend to leave aside the use of money (ingredient of economic calculation). However, implicitly assume the existence of money and its use, as talk (in general) of prices and costs, claiming confront cost prices. Prices are monetary magnitudes, and costs expressed in monetary terms only enter into economic calculation.

Other mathematical economists assert that economic calculation would be based on units of utility.

In Economics warned that people consciously want to bring about change. We know the forces driving change and such knowledge (based on reasoning) allows us to understand the economic process. The economist warns that what drives and causes the emergence of the market. With that knowledge fails to distinguish 


\section{Pablo Rivas Santos}

economic phenomena of physical phenomena may, by such a route, discover the laws governing the activities of the market.

In the field of monetary economics Microeconomics and negative effects are noticed mathematical analysis: The "quantity equation of money" is a barren and misguided attempt to address the problem of variations in the purchasing power of money. The statement "consumers, to assess consumer goods also value the factors of production necessary for the production of these consumer goods "is a false assumption to microecomico analysis.

The Economy is interested, directly by the people act. The only task of economic science is to analyze the actions of the people, that is, to analyze processes.

Keywords: Mathematical method, price statistics, economics, prices, costs.

\section{INTRODUCCIÓN}

Las cuestiones que suscitan los precios y costos se han pretendido abordarlos con arreglo a métodos matemáticos. Los economistas matemáticos decían que dicho sistema era el único apropiado para atacar los problemas económicos.

Si el antagonismo entre economistas lógicos y economistas matemáticos fuese un desacuerdo en cuanto al método más fecundo para estudiar Economía, sería ocioso prestar demasiada atención al asunto. El mejor método de ambos sistemas acreditaría su superioridad; al proporcionar mejores resultados. Incluso tal vez conviniera recurrir a diversos métodos según la clase de problema económico abordado.

Sin embargo, no estamos, ante cuestiones de métodos; la controversia se refiere al fundamento mismo de la Economía. El método matemático es un sistema que parte de falsos supuestos y conduce a erróneas conclusiones. Sus argumentos [ los cuales constan de 3 supuestos (el último supuesto se deduce de los otros dos) ] son inútiles y distraen la atención de los verdaderos problemas económicos, deformando la cadena entre los diversos fenómenos económicos.

Ni las ideas sustentadas ni los métodos usados por los economistas matemáticos son uniformes. Existen 3 escuelas: 


\section{Pensamiento Crítico Vol. I8. Nº}

En la primera escuela están los estadísticos, que aspiran a descubrir leyes económicas en base al análisis de la experiencia económica. Pretenden transformar la Economía en una ciencia cuantitativa.

\section{LOS PRECIOS}

La Estadística de precios es una forma de representar realidades históricas referentes a Precios. La Estadística de Precios es historia económica.

El Teorema económico: (manteniendo constante las restantes circunstancias) un aumento de la demanda provoca un alza del precio; no deriva de la experiencia. Nadie está en condiciones de observar el cambio (manteniendo constante las restantes circunstancias) de cierta circunstancia de mercado. Todas las magnitudes económicas son datos de historia económica. No existe relación constante entre demanda y precio (ni aun en lo relacionado a específicos bienes).

Por el contrario: los fenómenos externos influyen distintamente en las distintas personas; varía la reacción de un mismo individuo ante idéntico fenómeno externo; y no es posible clasificar a las personas en grupos de personas con idénticas reacciones. Estas verdades las deducimos de la Teoría económica basada en el razonamiento.

Los estadísticos dicen que ellos obtienen sus conocimientos de la experiencia histórica. Sin embargo, contradicen, sus propios principios tan pronto como (al pretender superar la anotación imparcial de precios singulares y específicos) comienzan a formular series y a calcular promedios. Lo único que la experiencia histórica nos dice y lo único que la estadística recoge, es determinado precio pagado en específico lugar y fecha por cierta cantidad de específico bien. Formular series con esos precios, y deducir promedios de esos precios, implica basarse en reflexiones teóricas, las cuales, lógica y temporalmente, anteceden a esas operaciones. El que en mayor o menor grado se tomen o no en consideración detalles acompañantes y contingencias circunstanciales que concurren con el precio, depende de un razonamiento teórico. Nadie ha afirmado que un aumento de $\mathrm{A} \%$ en la oferta de cierto bien forzosamente -en todo país y en todo tiempo- provocaría una reducción de $\mathrm{B} \%$ en su precio. Siendo así que ningún economista cuantitativo se atrevió a precisar concretamente (amparándose en la experiencia estadística) las específicas circunstancias que hacen variar la razón A\%: 


\section{Pablo Rivas Santos}

B\%. La inutilidad del sistema es evidente. Por otra parte, el dinero no es una unidad invariable que permita medir los precios; el dinero es un medio cuya razón de cambio también varía, con menor celeridad y amplitud que con la celeridad con que varía la razón recíproca de intercambio de bienes.

Los economistas cuantitativos se dedicaron a medir la elasticidad de la demanda de bienes. Los economistas cuantitativos jamás intentaron determinar la elasticidad de demanda de ningún bien, como tal; los datos que manejaban se refería sólo a cierta área geográfica y determinado período histórico. Sus estudios sobre determinado bien, las papas, por ejemplo no se refieren a las papas en general, sino a las papas de una localidad, y en la época comprendida entre determinados meses. Tales datos son contribuciones incompletas y discutibles a la historia económica. Las otras 2 escuelas de economía matemática advierten la esterilidad del método cuantitativo. Esas 2 escuelas nunca se han atrevido a operar ( en sus fórmulas y ecuaciones ) con magnitudes como las magnitudes halladas por los estadísticos, usando esas fórmulas y ecuaciones en la solución de problemas concretos. En el campo del actuar de la gente hay instrumentos idóneos para abordar eventos futuros como aquellos instrumentos que proporciona la Comprensión.

\section{LA RELACIÓN DE PRECIOS Y COSTOS}

Otro campo en que los economistas matemáticos se han interesado es el campo de la relación de precios y costos. Al abordar estos asuntos, ignoran la operación del mercado y pretenden dejar de lado el uso del dinero (ingrediente del cálculo económico). Sin embargo, implícitamente, suponen la existencia del dinero y su uso, ya que hablan (en general) de precios y costos, pretendiendo confrontar los precios con los costos. Los precios son magnitudes dinerarias; y los costos sólo expresados en términos monetarios entran en el cálculo económico. En otro caso, los costos habrán de computarse en cantidades formadas por los bienes de producción que es necesario emplear para obtener cierto bien de consumo. Tales precios -si es que cabe aplicar tal término a los tipos de cambio generados por el trueque- son simple enumeración de distintas cantidades de distintos bienes por los cuales el "vendedor" intercambia el específico bien que ofrezca. Los bienes a que tales precios se refieren; no son los mismos bienes que aquellos a los que los "costos" se referían. Por tanto, no es posible comparar entre si precios y costos en especie. Que el vendedor valora en menos los bienes entregados 


\section{Pensamiento Crítico Vol. I8. Nº}

que los bienes que recibe a cambio; que el vendedor y comprador discrepan en lo que respecta a la subjetiva valoración de esos dos productos cambiados; y que el empresario se lanza a determinada operación sólo cuando por el correspondiente producto a entregar espera recibir bienes mayormente valorados; que aquellos bienes empleados en la obtención del producto a entregar, eso ya lo sabíamos gracias a la Comprensión de la Economía. Tal conocimiento basado en el razonamiento nos permite prever la conducta que adoptará el empresario; cuando pueda recurrir al cálculo económico. El economista matemático se engaña al pretender abordar de un modo más general los problemas, omitiendo toda referencia a las expresiones monetarias. Porque es inútil pretender investigar las cuestiones que causa la divisibilidad imperfecta de los factores de producción; sin referirse al cálculo económico en términos monetarios. Tal análisis no proporciona más conocimientos que los conocimientos ya poseídos: que todo empresario procura producir aquellos bienes cuya venta piensa que le reportará ingresos valorados en más que el conjunto de bienes empleados en su producción. En ausencia del intercambio indirecto y del medio de cambio; ese empresario logrará su propósito, siempre y cuando haya anticipado correctamente la futura situación del mercado (sólo si disfruta de sobrehumana inteligencia). Tendría que: darse cuenta de golpe de cuantas razones de intercambio registraba el mercado; y valorar correctamente (con arreglo a esas razones de intercambio) los bienes que él mismo estaba manejando.

Toda investigación relacionada a la relación de precios y costos; supone el mercado y el uso del dinero. Sin embargo, los economistas matemáticos formulan ecuaciones y trazan curvas que, dicen, reflejan la realidad. Tales hipótesis se refiere a una situación imaginaria e irrealizable, sin parecido con los problemas de la Microeconomía. Se sirven de símbolos algebraicos (en vez de las expresiones monetarias empleadas en el cálculo económico) creyendo que así sus razonamientos son más científicos. Impresionan a economistas inexpertos; pero, lo que hacen es confundir y embrollar temas claros, que abordan los libros de contabilidad y matemática financiera.

\section{LA ANALOGÍA DE LA ECONOMÍA CON LA FÍSICA}

Algunos economistas matemáticos afirman que cabría basar el cálculo económico en unidades de utilidad. Denominan análisis de la utilidad a este sistema. En el mismo error incide el tercer grupo de los economistas matemáticos. 


\section{Pablo Rivas Santos}

Lo característico de este tercer grupo consiste en que pretenden resolver los problemas de la Microeconomía sin hacer referencia al proceso del mercado. Su ideal está en formular la teoría Microeconómica con arreglo al patrón de la mecánica física. Buscan analogías con la mecánica física. No es necesario insistir: en por qué tales analogías son accidentales y sólo sirven para inducir al error; ni insistir en las diferencias que separan el consciente actuar de la gente; del movimiento físico (objeto de investigación de la mecánica física ). Bastará con llamar la atención sobre un punto: el distinto significado práctico que tienen las ecuaciones diferenciales en la Física y la Microeconomía.

Los pensamientos que generan una ecuación diferencial son de carácter no matemático. En la ecuación diferencial se encarna anterior conocimiento; dicha expresión matemática, directamente, no amplia nuestro saber. No obstante ello, en el campo de la mecánica física las ecuaciones diferenciales han prestado importantes servicios. Ya que las relaciones que existen entre los factores físicos manejados son constantes y cabe comprobar experimentalmente dichas relaciones; es posible utilizar ecuaciones diferenciales para resolver problemas técnicos. La modernidad es fruto de recurrir a las ecuaciones diferenciales, en la física matemática. En cambio, entre los factores económicos, no hay relaciones constantes. Las ecuaciones diferenciales formuladas por la economía matemática es una inútil gimnasia mental y, aun cuando nos dijeran mucho más de lo que expresan, no por ello serían de mayor fecundidad.

El análisis económico considera 2 Principios de la Teoría del Valor: toda valoración generadora de acción implica preferir una cosa y rechazar otra cosa; y las valoraciones de diferentes personas (o las valoraciones de una misma persona, en momentos distintos) sólo se conocen contemplando cómo efectivamente la persona reacciona ante determinada alternativa.

En el modelo de una economía de rotación uniforme, todos los factores de producción están siendo empleados de tal suerte que cada uno de ellos rinde el servicio más valioso que puede proporcionar. No cabe pensar en modificación a cuyo amparo mejoraría el grado de satisfacción; ningún factor de producción se dedica a satisfacer la necesidad A si tal empleo impide satisfacer la necesidad B (de mayor valor que A). Cabe plasmar en ecuaciones diferenciales esta imaginaria distribución de factores de producción, así como darle gráfica representación mediante las correspondientes curvas. Todo ello nada nos dice del proceso del mercado. Estamos ante la descripción de una 


\section{Pensamiento Crítico Vol. I8. N I}

imaginaria distribución de factores de producción (así como darle gráfica representación mediante las correspondientes curvas). Estamos ante la descripción de una imaginaria situación que, implantada, paralizaría el proceso del mercado.

La Física se ocupa de cambios que registran los sentidos. Nos damos cuenta de una regularidad en la secuencia de esos cambios y tales observaciones nos permiten estructurar la Teoría Física. Sin embargo, nada sabemos de las fuerzas originarias que provocan esos cambios. Esos cambios son para el investigador, datos últimos que marginan todo posterior análisis. La observación nos permite apreciar la regular cadena existente entre diferentes fenómenos y circunstancias perfectamente observables. Esa mutua interdependencia entre los datos recogidos; es lo que el físico refleja mediante sus ecuaciones diferenciales.

En Economía advertimos que las personas conscientemente desean provocar cambios. La Economía se articula en torno a ese conocimiento; diferenciándose de la Física, en razón a esa circunstancia. Conocemos las fuerzas que provocan el cambio y tal conocimiento (basado en el razonamiento) nos permite comprender el proceso económico. El economista advierte cuál es eso que impulsa y provoca la aparición del mercado. Gracias a ese conocimiento logra distinguir los fenómenos sociales, de los demás fenómenos; pudiendo, por tal vía, descubrir las leyes rectoras de la actividad del mercado.

De ahí que la economía matemática no contribuye a dilucidar el proceso del mercado; porque solo describe simple modelo auxiliar que los economistas lógicos estructuran como concepto límite (aquella situación bajo la cual el actuar de la gente se esfumaría, quedando paralizado el mercado). Eso es, lo único, que nos habla traduciendo al lenguaje algebraico (lo que el economista lógico expone mediante lenguaje común) al establecer los supuestos de los imaginarios modelos de la situación final de reposo y de la economía de rotación uniforme; aquello mismo que el propio economista matemático se ve obligado a expresar, mediante lenguaje común, antes de comenzar a montar sus operaciones matemáticas, quedando todo, después, empantanado en simple figuración de poco valor.

Los economistas lógicos y matemáticos, reconocen que el actuar de la gente tiende a instaurar un estado de equilibrio que se alcanzara (si no se produjeran ya más cambios 


\section{Pablo Rivas Santos}

en las circunstancias concurrentes). Sin embargo, los economistas lógicos saben además de qué modo el actuar de personas emprendedoras, promotoras y especuladoras (ansiosas de lucrarse con las diferencias que registra la estructura de los precios) aboga por la eliminación de esas diferencias y, consecuentemente, por la obstrucción de la fuente que genera la ganancia y la pérdida empresarial. Demuestran cómo ese proceso evolucionaría hasta, finalmente, instaurar una economía de rotación uniforme. Tal es el cometido propio de la teoría económica. La matemática descripción de diversas situaciones de equilibrio es simple juego; lo que interesa es el examen y la comprensión del proceso del mercado.

La mutua contrastación de ambos sistemas de análisis económico; nos permite comprender mejor la esencia de aquella petición que postula la ampliación del ámbito de la Ciencia Económica mediante la elaboración de una teoría dinámica; abandonando la contemplación de los problemas estáticos. En lo que se refiere a la Economía Lógica, esa petición carece de sentido. La Economía Lógica es una teoría que examina procesos y cambios. Recurre a inmóviles e imaginarios modelos; para aprehender mejor el fenómeno del cambio. Pero, en lo referente a la economía matemática, la cosa es distinta. Las ecuaciones y fórmulas que maneja la economía matemática se limita a describir situaciones de equilibrio e inacción. Mientras no abandonen el terreno matemático, dichos investigadores nada pueden decirnos acerca de la génesis de tales situaciones ni de cómo esas situaciones pueden evolucionar y dar lugar a distintos planteamientos. En lo que se refiere a la economía matemática, la petición de una teoría dinámica está plenamente justificado. Sin embargo, la economía matemática carece de medios para satisfacer tal petición. Los problemas que suscita el análisis del proceso del mercado son imposibles de abordar por medios matemáticos. La introducción de parámetros temporales en las correspondientes ecuaciones de nada sirve. Ni siquiera se roza con ello las deficiencias fundamentales del método matemático. El decir: que todo cambio requiere cierto período de tiempo; y que el cambio implica, en todo caso, secuencia temporal es otro modo de decir que, donde haya rigidez e inmovilidad absoluta, el factor tiempo desaparece. El defecto de la economía matemática no está en ignorar la secuencia temporal, sino en que da la espalda al funcionamiento del proceso del mercado. 


\section{Pensamiento Crítico Vol. 18. N I}

\section{CONCLUSIONES}

El método matemático no puede explicar cómo en cierto estado de desequilibrio surge aquel actuar de la gente que tiende a generar el equilibrio. Cabe indicar la serie de operaciones matemáticas necesaria para transformar la descripción matemática de cierto estado de desequilibrio en la descripción matemática del estado de equilibrio. Esa serie de operaciones no refleja el proceso del mercado que pone en marcha las discrepancias en la estructura de los precios. En la mecánica física, se admite que las ecuaciones diferenciales retratan con precisión las diversas situaciones sucesivamente registradas durante el tiempo. Sin embargo, las ecuaciones económicas no reflejan las diferentes circunstancias propias de cada instante comprendido en el intervalo temporal que separa el estado de desequilibrio del estado de equilibrio.

En el campo de la Economía se advierte los perniciosos efectos del análisis matemático. Sean dos ejemplos. El primer ejemplo nos brinda la "ecuación cuantitativa del dinero"; ese estéril y errado intento de abordar el problema de las variaciones del poder adquisitivo del dinero. El segundo ejemplo el cual afirma "que los consumidores, al valorar los bienes de consumo, valoran también los factores de producción necesarios para la obtención de esos bienes de consumo".

La Economía se interesa, directamente por el actuar de las personas. El único cometido de la Ciencia Económica consiste en analizar el actuar de la gente, o sea, en analizar procesos.

\section{REFERENCIAS BIBLIOGRÁFICAS}

Friedman, Milton (1971). Nueva reformulación de la teoría cuantitativa del dinero. España. Editorial Continental. Primera Edición. Pag. 123-152.

Mundell, Robert A. (1971). Teoría monetaria. España. Ediciones Amorrurtu Editores. Pag. 323-430.

Von Mises Ludwig (1966). Teoría de la moneda y el crédito. España. Fundación Ignacio Villalonga. Pag. 239-452.

Von Mises Ludwig (1980). Acción humana. España. Fundación Ignacio Villalonga. Pag. 180-332. 